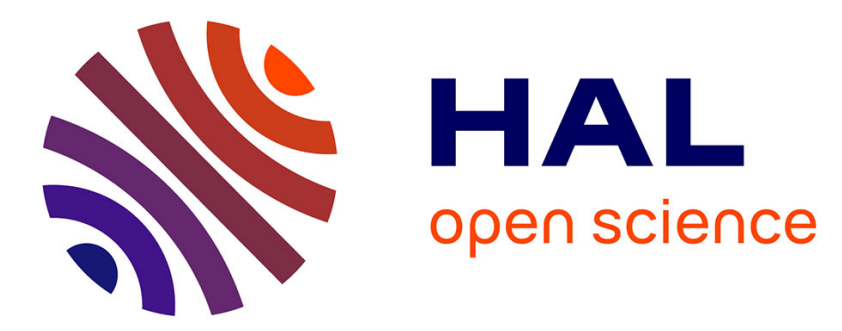

\title{
High-resolution-scanning waveguide microscopy: spatial refractive index and topography quantification
}

L. Berguiga, R. Orobtchouk, J. Elezgaray, Alain Arneodo, Françoise Argoul

\section{To cite this version:}

L. Berguiga, R. Orobtchouk, J. Elezgaray, Alain Arneodo, Françoise Argoul. High-resolution-scanning waveguide microscopy: spatial refractive index and topography quantification. Optics Letters, 2017, 42 (13), pp.2523 - 2526. 10.1364/OL.42.002523 . hal-01548509

\author{
HAL Id: hal-01548509 \\ https://hal.science/hal-01548509
}

Submitted on 27 Jun 2017

HAL is a multi-disciplinary open access archive for the deposit and dissemination of scientific research documents, whether they are published or not. The documents may come from teaching and research institutions in France or abroad, or from public or private research centers.
L'archive ouverte pluridisciplinaire HAL, est destinée au dépôt et à la diffusion de documents scientifiques de niveau recherche, publiés ou non, émanant des établissements d'enseignement et de recherche français ou étrangers, des laboratoires publics ou privés.

\section{(1)(1) $\$(0)$}

Distributed under a Creative Commons Attribution - NonCommercial - ShareAlikel 4.0 


\title{
High-resolution-scanning waveguide microscopy: spatial refractive index and topography quantification
}

\author{
L. Berguiga, ${ }^{1, *}$ R. Orobtchouk, ${ }^{1}$ J. Elezgaray, ${ }^{2}$ A. Arneodo, ${ }^{3,4}$ and F. Argoul ${ }^{3,4,5}$ \\ ${ }^{1}$ Institut des Nanotechnologies de Lyon (INL), CNRS UMR5270, Université de Lyon, INSA-Lyon, 7 avenue Jean Capelle, \\ Villeurbanne F-69621, France \\ ${ }^{2}$ CNRS UMR 5248, CBMN, Université de Bordeaux, Allée Geoffroy Saint Hilaire, Bât B14, 33600 Pessac, France \\ ${ }^{3}$ CNRS UMR 5798, LOMA, Université de Bordeaux, 351 Cours de la Libération, 33405 Talence, France \\ ${ }^{4}$ CNRS UMR 5672, Laboratoire de Physique, ENS Lyon, Université de Lyon, 46 Allé d'Italie, 69007 Lyon, France \\ ${ }^{5}$ e-mail: francoise.argoul@u bordeaux.fr \\ *Corresponding author: lotfi.berguiga@insa lyon.fr
}

\begin{abstract}
We report on a high-resolution metal-clad waveguide scanning microscopic method with a diffraction-limited resolution. This microscope can be operated in both TM and TE waveguide modes with radially and azimuthally polarized beams, respectively, and allows both refractive index and topography of dielectric objects to be evaluated at high resolution and sensitivity. We emphasize the performance of this microscopic method from calibrated 3D polymer microstructures with rectangular, disk, and ring shapes.
\end{abstract}

OCIS codes: (180.3170) Interference microscopy; (110.6960) Tomography; (130.2790) Guided waves; (240.6680) Surface plasmons.

https://doi.org/10.1364/OL.42.002523

Owing to their ability to amplify reflected electromagnetic fields, surface plasmon resonance (SPR) and dielectric wave guides (WGs) have revolutionized free label optical biosensing techniques [1]. Both methods rely on evanescent wave excita tion, the depth of which ( 200 nm in the visible spectrum) allows separation of the optical response of the sample interface from the bulk. On the other hand, metal clad waveguides (MCWGs) allow the exploration of regions distant by several microns from the interface, with the additional interest of nar rowing the resonance width [2 5$]$. They are composed of a thin layer of metal (several tens of nanometers) and a thick dielectric layer (several hundreds of nanometers) that support the MCWG modes (MCWGMs). MCWGs are also called plas mon resonance waveguides (PRWGs), coupled plasmon reso nance waveguides (CPRWGs), or metal clad leaky waveguides (MCLWGs). A prism coupled MCWG is limited in resolution by a few micrometer size decay of the lateral propagating guided wave. A first version of a MCWG microscope with a lateral resolution $<10 \mu \mathrm{m}$, collecting the reflected light, was proposed in 1990 [6]. Interference between the guided wave with its reflections on the border of a finite size index step can smear the guided wave outside the step [7] and deteriorate the resolution and image contrast, requiring further image processing [8]. Söllradl et al. [9] proposed to couple the guided wave with a high numerical aperture microscope objective lens; however, the lateral resolution (along the $x$ axis) of their wide field microscope remained limited by the lateral decay propa gation length of the MCWG (several microns). Scanning surface plasmon microscopy (SSPM) uses a strongly focused incident beam to reach the diffraction limit [10 13]. SSPM can also be coupled to the MCWG to measure refractive index and thickness of $1 \mathrm{D}$ dielectric slabs [14]. In this Letter, we re port the performance of SSPM for MCWGM imaging of di electric objects such as three dimensional (3D) polymer microstructures with high resolution (FWHM $=180 \mathrm{~nm}$ for $\lambda=632.8 \mathrm{~nm}$ ), in both TE and TM light beam polariza tions. We show that tomographic reconstruction of these 3D dielectric structures can be achieved at submicrometric res olution in the lateral directions $(x, y)$, nanometric precision in the $z$ direction, and two per thousand precision for the refractive index. We also provide numerical computations of the MCWGs from $1 \mathrm{D}$ and $2 \mathrm{D}$ models that validate the MCWGMs detected experimentally with SSPM.

The SSPM principle and optical setup are sketched in Fig. 1. MCWGMs were excited with a high numerical aperture $(\mathrm{NA}=1.57)$ objective lens. When the objective lens was fo cused beyond the gold film $(z>0)$, MCWGMs propagated at the gold/dielectric interface symmetrically toward the objective axis and were partly reflected to the back focal plane of the ob jective lens by radiation leakage (RL) [Fig. 1(a)]. Two kinds of reflected rays contributed to the back reflected light: leaking rays (from RL) from the excited MCWGMs and other reflected rays that did not contribute to MCWGMs. A phase difference between these two kinds of rays occurred and due to MCWGM propagation this phase varied with the defocusing. This phase difference provided information about MCWGM excitation conditions in the focused region and was assessed by hetero dyne interferometric detection. As MCWGMs can be excited 
separately with TE and TM light polarizations, azimuthally and radially polarized beams were used to excite independently these two kinds of modes. Actually the electric field near the focus has a purely longitudinal component (in $z$ direction) for a radially polarized beam and a purely transverse component (in $x$ and $y$ directions) for an azimuthally polarized beam. A liquid crystal cell polarization converter (PC) was used to convert a linearly polarized beam into a radially or azimuthally polarized beam and to record images in TM or TE polarization separately. The $V(z)$ optical response of this microscope versus the defocus $z$ can be obtained as an integral of the reflected electric field [10]:

$$
V(z) \propto \int_{0}^{2 \pi} \int_{0}^{\theta_{\max }} P^{2}(\theta) R(\theta) e^{i 4 \pi n_{1} z \cos \theta / \lambda} \sin \theta \mathrm{d} \theta \mathrm{d} \varphi,
$$

where $P(\theta)$ is the pupil function of the objective lens, $\theta$ and $\varphi$ are the polar and azimuthal angles, $n_{1}$ is the refractive index of the coupling medium (the objective lens, the cover slip, and the immersion oil), and $R(\theta)$ is the complex reflection coefficient of the sample. $R_{\mathrm{TM}}(\theta)$ (resp. $R_{\mathrm{TE}}(\theta)$ ) were obtained with a purely TM (resp. TE) polarization using a radially (resp. azi muthally) polarized beam. With the change of variable $\nu=2 n_{1} \cos (\theta) / \lambda, \quad R(\theta) \cdot P^{2}(\theta)$ can be recovered by a Fourier transform of $V(z)$ [14]. Resonant MCWGMs are char acterized by strong absorption at specific incident angles, trans lating into narrow dips of the $R(\theta) \cdot P^{2}(\theta)$ curves.

At each position $(x, y, z)$ of the piezoelectric stage and time $t$, the recorded demodulated field on the detector is propor tional to the so called $V(x, y, z, t)$ response function:

$$
I_{\mathrm{SSPM}}(x, y, z, t) \propto \sqrt{ } I_{1} I_{2}(x, y, z, t) e^{i \psi(x, y, z, t)} \propto V(x, y, z, t),
$$

where $I_{1}$ (resp. $I_{2}$ ) is the optical intensity of the light coming from the first (resp. second) arm of the interferometer. For a fixed $(x, y)$ position, a $V(z)$ curve was reconstructed from a

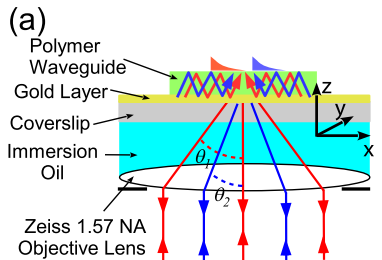

(b)

(c)

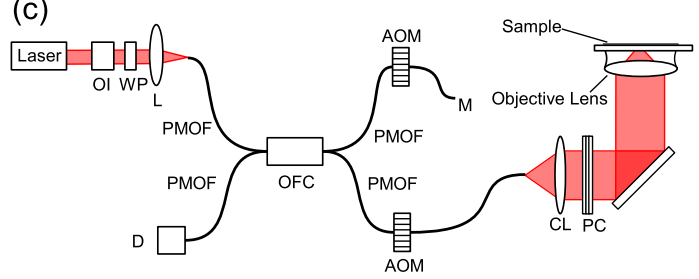

Fig. 1. Scanning surface plasmon microscope operation mode. (a) Sample configuration with the MCWG excitation through a $100 \times /$ NA 1.57 Carl Zeiss objective lens. (b) Spatial distribution of the polarization at the entrance of the objective lens aperture. The PC switches between a radially and an azimuthally polarized beam. (c) Microscope setup including a fibered heterodyne interferometer, $\mathrm{He} \mathrm{Ne}$ laser $(632.8 \mathrm{~nm})$; OI, optical isolator; WP, half wavelength waveplate; L, lens; PMOF, polarization maintaining optical fiber; OFC, optical fiber coupler; AOM, acousto optic modulator; D, de tector; CL, collimating lens; $\mathrm{M}$, mirror. $z$ scanning. For a given $z^{*}$ position, $500 \times 500$ pixel images were recorded by 2D scanning along the $x$ and $y$ directions. Before each image acquisition, a single $V_{\text {ref }}(z)$ curve was re corded at the first pixel of the image for calibration and sample tilt correction [15]. The raw image modulus $I_{R}\left(x, y, z^{*}, t\right)=$ $\left|I_{\text {SSPM }}\left(x, y, z^{*}, t\right)\right|$ was normalized by its maximum value $\left|V_{\text {ref }}(z=0)\right|$, and a computed background intensity map $I_{B}(x, y, t)$ was subtracted to obtain the corrected image $I_{C}(x, y)[12]$, as follows:

$$
I_{C}(x, y, t)=\frac{I_{R}(x, y, t) \quad I_{B}(x, y, t)}{\left|V_{\text {ref }}(z=0)\right|} .
$$

The investigated polymer structures $(30 \times 5 \mu \mathrm{m}$ rectangles, $15 \mu \mathrm{m}$ diameter disks, and rings with $15 \mu \mathrm{m}$ [resp. $13 \mu \mathrm{m}$ ] outer [resp. inner] diameters) were deposited onto a $45 \mathrm{~nm}$ gold layer anchored on a high refractive index (1.648) glass coverslip via a $1.5 \mathrm{~nm}$ chromium layer.

Figures 2(a) and 2(b) report the modulus and the phase of $V(z)$ curves recorded for TM (a) and TE (b) light polarization, on two positions, $A$ and $B$, selected, respectively, on a naked gold surface (SPR black curves) and polymer structure (MCWG red curves) [see also Figs. 4(a) and 4(c)]. With TM light polari zation [Fig. 2(a)] both $V(z)$ curves present periodic oscillations for positive $z$ values. For positive $z$ values the waveguide modes converge toward the center, whereas for negatives ones they di verge from the center and, consequently, no standing waves are formed. With TE light polarization [Fig. 2(b)], only the $V(z)$ curve collected from the polymer structure shows periodic oscil lations. The difference between $V(z)$ curves recorded at posi tions $A$ and $B$ explains the intensity modulation of the image obtained with this microscope for each polarization. The modu lus and phase of $R(\theta) \cdot P^{2}(\theta)$ were computed from the $V(z)$ curves for the same positions [Figs. 2(c) and 2(d)]. On the bare gold surface (black curves, position A), a resonance dip appears on $R(\theta)$ in TM polarization only [Fig. 2(c)] corresponding to SPR excitation. On the polymer structure, two resonance dips, corresponding to two MCWGMs, occur in both TM [Fig. 2(c)] and TE [Fig. 2(d)] polarizations.

As a first approximation, we fitted the resonance dip posi tions of the polymer structure in TM mode [Fig. 2(a)] with a
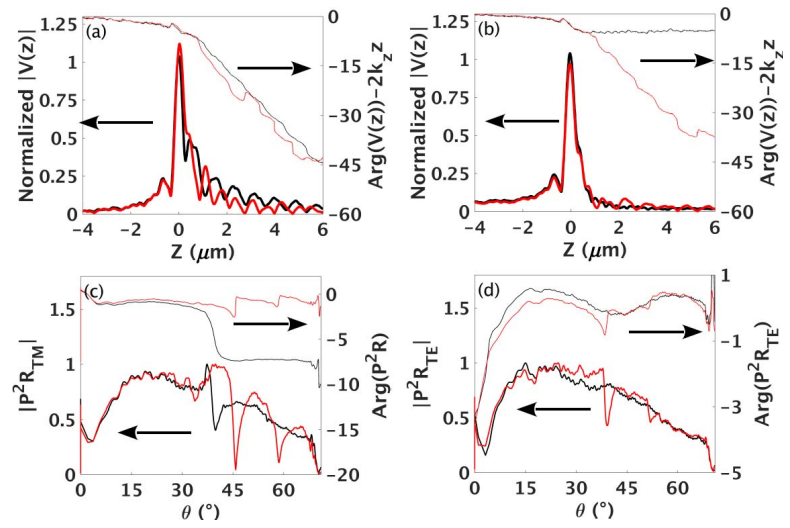

Fig. 2. Modulus and phase of the $V(z)$ curves recorded with TM (a) and TE (b) polarized light from two positions, $A$ (black curve naked gold film) and $B$ (red curve rectangular polymer structure) pointed by arrows in Figs. 4(a) and 4(c). Modulus and phase of $R(\theta)$. $P^{2}(\theta)$ computed from the $V(z)$ curves for the same positions, with TM (c) and TE (d) polarized light beams. 
one dimensional (1D) Fresnel equation model (four infinite layers: glass gold dielectric bulk medium), and we estimated its thickness and refractive index to be $\sim 732 \mathrm{~nm}$ and 1.478 , respectively. These two parameter values were used to elaborate a two dimensional (2D) infinite rail structure (along $y$ ) of width $5 \mu \mathrm{m}$ along the $x$ direction from which MCWGMs were cal culated with a homemade full vectorial finite difference mode solver [16]. This infinite rail is a fair approximation of the pol ymer rectangle pattern in Fig. 4(a). Effective indices (defined as $n_{\text {eff }}=\beta \lambda / 2 \pi$ where $\beta$ is the propagation constant of the wave guide) and mode profiles were obtained by solving a system of two coupled eigenvalue equations for the TE or TM field components of the guided wave [16]. We used a nine point discretization scheme to described accurately the electric dis continuity at the boundary interfaces of the structure without the need of a graded index approximation. Details of the algo rithm are given in Ref. [16]. Transparent boundary conditions were also implemented to consider leakages through the gold coated coverslip substrate. The MCWGMs that appear in the $2 \mathrm{D}$ structures are confined in the $x z$ plane and propagate along the $y$ direction. The electric field overfills the structure within the $x z$ plane and is structured as bumps and nodes oriented along the $x$ and $z$ directions. The number of nodal lines in the $x$ direction corresponds to the order of the MCWGM (its level of degeneracy). The effective indices and the corre sponding angles of excitation of the modes are reported in Table 1. For each MCWGM order $(\mathrm{N})$, the first three degen erated modes $[\mathrm{N}, 0],[\mathrm{N}, 1]$, and $[\mathrm{N}, 2]$ corresponding to respec tively 0,1 , and 2 line nodes in the $z$ direction are reported. The effective indices and the angles of resonance dips of the zeroth line $[\mathrm{N}, 0]$ of the degenerated modes for each order (the field has no nodal line in the $z$ direction) computed with the $2 \mathrm{D}$ simulation match those obtained with the 1D simulation. This means that the propagating condition of the modes in the $y$ direction is the same for an infinite layer or a rail, either of which possesses the same thickness and refractive index. For the other degenerated modes ([N,1] and $[\mathrm{N}, 2]$ corresponding to 2 and 3 nodal lines), the effective index decreases with the

Table 1. Effective Indices and Excitation Angles of MCWGM Resonance Dips Obtained from 1D and 2D Simulations and Experiments in TM and TE Polarizations ${ }^{a}$

\begin{tabular}{|c|c|c|c|c|c|c|c|}
\hline & \multirow[b]{2}{*}{ Modes } & \multicolumn{2}{|c|}{ Theory (1D) } & \multicolumn{2}{|c|}{ Theory (2D) } & \multicolumn{2}{|c|}{ Experiment } \\
\hline & & $n_{\text {eff }}$ & $\theta\left({ }^{\circ}\right)$ & $n_{\text {eff }}$ & $\theta\left({ }^{\circ}\right)$ & $n_{\text {eff }}$ & $\theta\left({ }^{\circ}\right)$ \\
\hline \multirow[t]{6}{*}{$\mathrm{TM}$} & {$[1,0]$} & 1.395 & 57.83 & 1.395 & 57.83 & 1.399 & 58.09 \\
\hline & {$[1,1]$} & & & 1.391 & 57.57 & & \\
\hline & {$[1,2]$} & & & 1.384 & 57.12 & & \\
\hline & {$[2,0]$} & 1.165 & 44.98 & 1.167 & 45.08 & 1.164 & 44.94 \\
\hline & {$[2,1]$} & & & 1.163 & 44.86 & & \\
\hline & {$[2,2]$} & & & 1.155 & 44.48 & & \\
\hline \multirow[t]{8}{*}{$\mathrm{TE}$} & {$[1,0]$} & 1.431 & 60.26 & 1.430 & 60.33 & nd & nd \\
\hline & {$[1,1]$} & & & 1.426 & 59.91 & & \\
\hline & {$[1,2]$} & & & 1.419 & 59.43 & & \\
\hline & {$[2,0]$} & 1.283 & 51.13 & 1.284 & 51.18 & 1.285 & 51.24 \\
\hline & {$[2,1]$} & & & 1.279 & 50.90 & & \\
\hline & {$[2,2]$} & & & 1.276 & 50.74 & & \\
\hline & {$[3,0]$} & 1.022 & 38.24 & 1.027 & 38.55 & 1.017 & 38.11 \\
\hline & {$[3,1]$} & & & 1.021 & 38.28 & & \\
\hline
\end{tabular}

${ }^{a}$ For the $2 \mathrm{D}$ simulations the polymer structure was approximated by an infinite rail of width $5 \mu \mathrm{m}$ with the same thickness and refractive index as estimated from the 1D simulation (732 $\mathrm{nm}$ and 1.478 , respectively). nd: not detected. number of nodal lines. The effective refractive indices estimated from the experimental $V(z)$ curves (Table 1) remarkably agree with the first degenerated mode $[\mathrm{N}, 0]$ of each order $(2 \mathrm{D}$ or $1 \mathrm{D}$ simulations), suggesting this SSPM setup mainly detects this first degenerated mode. This effect can be explained by the cou pling efficiency of the objective lens being limited by the overlap between the incoming Bessel beam shape and the lateral profile of the guided wave that is maximum for the first degenerated mode and decreasing for the higher ones, in such a way that the related resonance dips are too small to be detected. We con clude from the comparison of $1 \mathrm{D}$ and $2 \mathrm{D}$ simulations with experimental data that an infinite layer simulation (1D) would be sufficient to predict our experimental measurements from 3D polymer structures. More surprisingly, the order 2 and 3 reso nance dips obtained experimentally in TE polarization are well fitted by both the 1D slab and 2D models even if the first order resonance dips were not detectable (Table 1). This resonance is too narrow to be detected. Actually, in the Fourier transform of the $V(z)$ curve, the $z$ range is limited to $120 \mu \mathrm{m}$ leading to convolution of the real reflectivity curve by a sinc function. Narrow resonance is smoothed out, becoming undetectable.

A $100 \times 45$ grid of $V(z)$ curves was recorded with a spacing of $485 \mathrm{~nm}$ in $x$ and $y$. Each $V(z)$ curve was inverted to recover the complex $R(\theta) P^{2}(\theta)$. Images of the number of resonance dips [Fig. 3(a)], of the MCWGM effective index for order 1 [Fig. 3(b)] and 2 [Fig. 3(c)] modes and of the estimated thick ness [Fig. 3(d)] and refractive index [Fig. 3(e)] of the polymer structure were reconstructed in $2 \mathrm{D}(x, y)$ for TM polarization. We can see that a single SPR is detected outside the polymer structure, whereas two MCWGMs are detected inside the pol ymer structure. Interestingly, at the boundary of the polymer structure a greater number of modes $(\gtrsim 4)$ can be detected. The thickness and the refractive index were estimated by fitting the resonance position in TM mode [Figs. 3(d) and 3(e)] with the $1 \mathrm{D}$ slab model. The rectangular structure is thicker in its center $(735 \mathrm{~nm})$ and thinner toward the borders $(710 \mathrm{~nm})$, whereas the refractive index appears more homogeneous all over the polymer structure with a value of $1.477 \pm 0.007$. This evaluation is very accurate since the refractive index value provided by the constructor for this polymer resin is 1.478 .

The corrected intensity images $I_{c}\left(x, y, z^{*}\right)$ obtained with SSPM for different polymer structures are shown in Fig. 4 for both TM (a, b, e, f, I, and j) and TE polarizations (c, d, $\mathrm{g}, \mathrm{h}, \mathrm{k}$, and l). The different polymer structures appear with a very high positive or negative contrast depending on the choice of scan height position $z^{*}$, mostly enhancing this
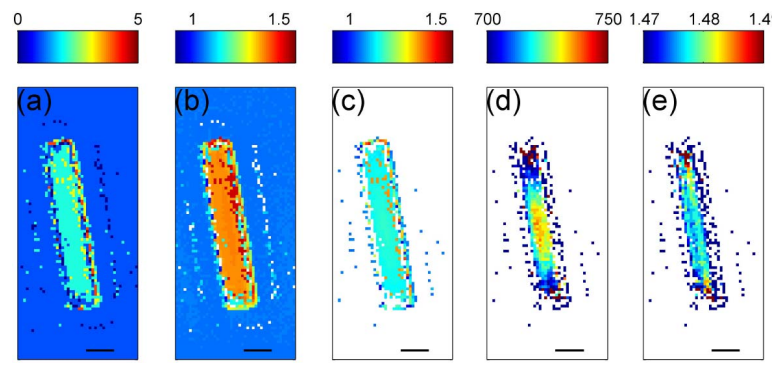

Fig. 3. Reconstructed MCWGM resonances from the grid of $V(z)$ curves in TM mode. (a) Number of detected resonances. (b), (c) Effective indices for order one and two mode resonances, respectively. (d), (e) Estimated thickness and refractive index inside the rectangular polymer structure. Scale bars: $5 \mu \mathrm{m}$. 
contrast. Sections of the rectangle, disk, and ring structures are represented in Figs. $4(\mathrm{~m})$ 4(o), respectively. The sizes of these structures were estimated from the TM polarization images at middle height as $29.2 \times 5.3 \mu \mathrm{m}$ for the rectangle, $10.1 \mu \mathrm{m}$ for the diameter of the disk and $12.1 \quad 15.3 \mu \mathrm{m}$ for the inner outer diameters of the ring. The sizes estimated from the TE polari zation images at middle height are slightly different: $28.4 \times$ $4.4 \mu \mathrm{m}$ for the rectangle, $9.54 \mu \mathrm{m}$ for the diameter of the disk and $12.714 .6 \mu \mathrm{m}$ for the inner outer diameter of the ring. These sizes are quite close to those found with scanning electron microscopy $(29.1 \times 5.03 \mu \mathrm{m}$ for the rectan gle, $10.12 \mu \mathrm{m}$ for the diameter of the disk, and 12.76 $15.33 \mu \mathrm{m}$ for the inner outer diameter of the ring). The MCWGM microscope can resolve dielectric structures as small as $1.3 \mu \mathrm{m}$ (the width of the ring). This width is actually over estimated $(1.6 \mu \mathrm{m})$ in TM polarization and underestimated $(0.95 \mu \mathrm{m})$ in TE polarization. The structures appear narrower in TE polarization than in TM polarization. This observation is compatible with the fact that the electromagnetic field of the MCWGMs in TE polarization does not fill up the whole struc ture. Importantly, we note that, compared to previous raw images collected from on prism coupled MCWGM [8], we do not observe with objective coupled MCWGM spurious oscillations outside the border of the structures. This can be explained by the greater sensitivity and resolution power of SSPM. For instance, the presence of some curvature on top of the rectangular structure was observed in TE as well as in TM polarizations [see sections in Figs. $4(\mathrm{~m})$ and $4(\mathrm{n})$ ]. This reinforces the power of this microscope for topographical im aging and reconstruction of 3D structure refractive index inho mogeneities at high spatial lateral resolution and nanometric precision. We also notice a darker (resp. brighter) annulus around the disk in TE polarization [Fig. 4(g) (resp. 4(h))] that is the signature of a whispering gallery mode. The excitation
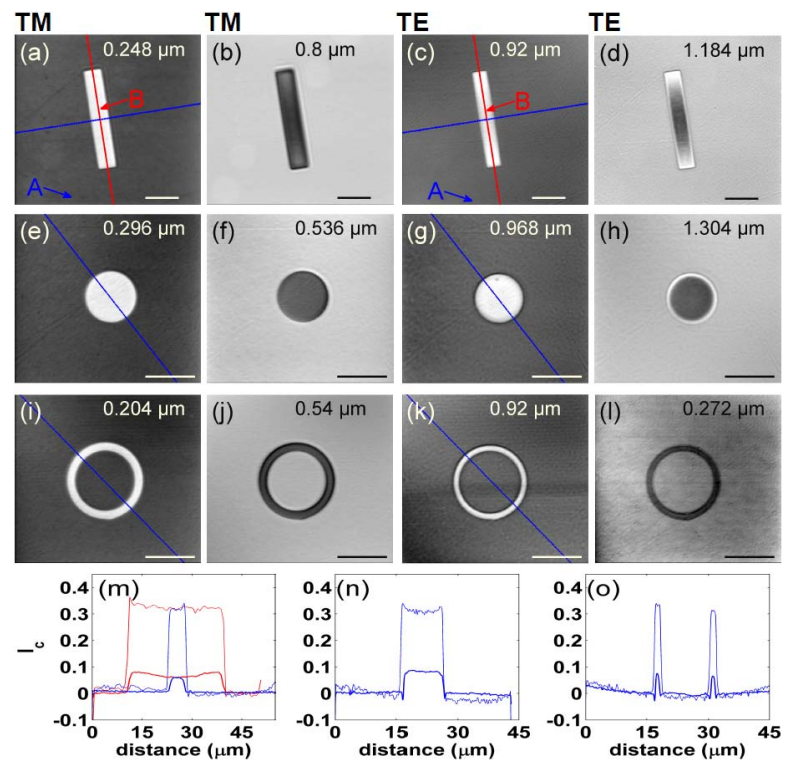

Fig. 4. MCWGM images in TM and TE polarization for rectan gular, (a) (d); disk, (e) (h); and annular, (i) (l) polymer structures. Scale bars: $10 \mu \mathrm{m}$. (m) (o) show the sections plotted as blue and red lines in the corresponding MCWGM images for the rectangular, disk, and ring structures, respectively. The thin (thick) lines corre spond to TM (TE) polarization. and the detection of whispering gallery modes are possible with SSPM. Moreover, the contrast of the whispering gallery mode or/and the MCWGM inside the disk structure can be tuned independently by changing $z^{*}$, the $x y$ scanning height. This $z$ tuning makes this microscopy method powerful. Actually compared to the method proposed in Ref. [9], no focusing of the resonance angle is needed. A complete study of the whis pering gallery mode imaging with SSPM in the ring and the disk will be reported in a forthcoming paper.

To summarize, we have shown in this study that MCGWM images can be reconstructed from $V(z)$ curves recorded with SSPM from 3D dielectric polymer structures. Given that we can recover the polymer structure characteristic sizes with both TM and TE polarizations, we conclude that the excitation and the detection of the MCWGMs occur at submicronic scale all over the polymer structures and lead to high resolution MCWGM imaging. To explain why this microscope appa rently detects only the first degenerated mode for each MCWGM order, we could argue that the light confinement by the objective lens could precisely select the modes with a minimum number of nodes (smoother field distribution). This microscope shows an unprecedented suitability to com bine a high spatial resolution and a high sensitivity for resolving the thickness and the refractive index of $3 \mathrm{D}$ complex dielectric structures deposited on a metal surface.

Funding. Agence Nationale de la Recherche (ANR) (ANR AA PPPP 005, EMMA 2011, IDEX 0007 02); Lyon Science Transfert (project L659); Région Rhône Alpes (CIBLE2011).

Acknowledgment. We are grateful to IEMN (UMR CNRS 8520 Av Poincaré 59652 Villeneuve d'Ascq) and especially to Isabelle Roch Jeune for the preparation of the structured samples.

\section{REFERENCES}

1. X. Fan, I. M. White, S. I. Shopova, H. Zhu, J. D. Suter, and Y. Sun, Anal. Chim. Acta 620, 8 (2008).

2. Z. Salamon, H. Macleod, and G. Tollin, Biophys. J. 73, 2791 (1997).

3. N. Skivesen, R. Horvath, S. Thinggaard, N. B. Larsen, and H. C. Pedersen, Biosens. Bioelectron. 22, 1282 (2007).

4. A. Lahav, M. Auslender, and I. Abdulhalim, Opt. Lett. 33, 2539 (2008).

5. F. Bahrami, M. Maisonneuve, M. Meunier, and J. S. Aitchison, Opt. Express 21, 20863 (2013).

6. W. Hickel and W. Knoll, Appl. Phys. Lett. 57, 1286 (1990).

7. B. Rothenhäusler and W. Knoll, J. Opt. Soc. Am. B 5, 1401 (1988).

8. F. Banville, T. Söllradl, P. J. Zermatten, M. Grandbois, and P. G. Charette, Opt. Lett. 40, 1165 (2015).

9. T. Söllradl, F. Banville, V. Chabot, M. Canva, M. Grandbois, and P. G. Charette, Opt. Express 25, 1666 (2017).

10. M. G. Somekh, S. G. Liu, T. S. Velinov, and C. W. See, Opt. Lett. 25, 823 (2000).

11. L. Berguiga, S. Zhang, F. Argoul, and J. Elezgaray, Opt. Lett. 32, 509 (2007).

12. L. Berguiga, L. Streppa, E. Boyer Provera, C. Martinez Torres, L.Schaeffer, J. Lezgaray, A. Arneodo, and F. Argoul, Appl. Opt. 55, 1216 (2016).

13. F. Argoul, L. Berguiga, J. Elezgaray, and A. Arneodo, Handbook of Photonics for Biomedical Engineering, A. H. P. Ho, D. Kim, and M. G. Somekh, eds. (Springer, 2016), pp. 142.

14. L. Berguiga, E. Boyer provera, C. Martinez Torres, J. Elezgaray, A. Arneodo, and F. Argoul, Opt. Lett. 38, 4269 (2013).

15. L. Berguiga, E. Boyer Provera, J. Elezgaray, and F. Argoul, Plasmonics 8, 715 (2013).

16. X. Hu, S. Cueff, P. R. Romeo, and R. Orobtchouk, Opt. Express 23, 1699 (2015). 